\title{
LARGE-AREA ELECTROSTATIC-VALVED SKINS FOR ADAPTIVE FLOW CONTROL ON ORNITHOPTER WINGS
}

\author{
Matthieu Liger, Nick Pornsin-Sirirak, Yu-Chong Tai \\ Caltech Micromachining Laboratory \\ Electrical Engineering Department, California Institute of Technology \\ Pasadena, CA 91125 \\ Steve Ho and Chih-Ming Ho \\ Mechanical and Aerospace Engineering \\ University of California \\ Los Angeles, CA 90095
}

\begin{abstract}
This paper presents the design and fabrication of wafer-sized parylene skins with integrated electrostatic valves used for realtime adaptive airflow control on ornithopter wings. For the first time, we report successful aerodynamic control in both lift and thrust using MEMS active-valved wings. By distributing electrostatic valves, the pressure distribution on the wings can be controlled during flapping. The valved wings were tested in a lowspeed wind tunnel to measure influence on aerodynamic performance. It was found the lift and thrust generated by the wings in flapping motion can increase by $18 \%$ when the valves are actuated.
\end{abstract}

\section{INTRODUCTION}

In a previous study in our group, MEMS-technology parylene wings with titanium alloy were fabricated and tested in flapping motion in a low-speed wind tunnel [1]. Aerodynamic study of the flapping-flight shows that performance is highly dependent on the formation of the unsteady leading-edge vortex. Therefore, pressure distribution control on the wings can be used to achieve optimal aerodynamic performance. Distributed valve actuators on the wings can provide such control. This concept is illustrated in Figure 1. In order to be integrated on flexible parylene MEMS wings, these valve actuators need to be light and flexible as well. Thus, we developed a wafer-sized parylene actuator "skins" technology. In [2] we used that technology to demonstrate that the performance of flapping flight can be greatly increased by integrating "passive" check-valves onto the parylene wings. The ultimate goal in our study, hence this work is to fabricate active valved-skins to dynamically control the wing's pressure loading. In this paper, we present a flexible electrostatic valved-skin using parylene technology. Aerodynamic test results of valved-wings will be discussed, as well as encountered challenges and possible future work.

\section{DESIGN AND FABRICATION}

The design and process-flow of the electrostatic valved-skins is based on a low-temperature multi-layer parylene technology incorporating metal electrodes. The skins are made of 4 parylene layers with sandwiched metal electrodes between the first and

Travel support has been generously provided by the

Transducers Research Foundation and by the DARPA MEMS and DARPA BioFlips programs

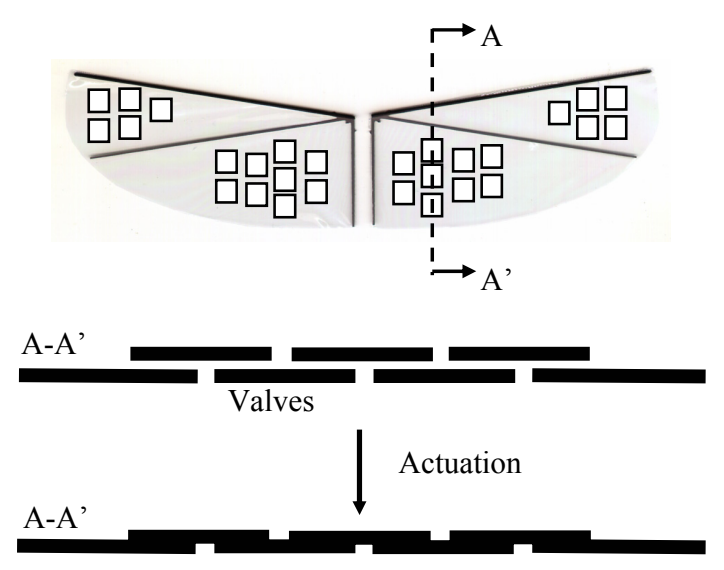

Figure 1: Integrated actuator MEMS wings concept

second layers and between the third and fourth layers. The valves feature $500 \mu \mathrm{m}$ diameter vent-holes that can be closed by tethered valve caps. The vent-holes are $1.28 \mathrm{~mm}$ apart from each other.. Square-shaped valve caps were used in order to maximize the electrostatic actuation area. The square caps are $1.02 \mathrm{~mm}$ long. When no voltage is applied between the top and bottom electrodes, the valves act like passive check-valves. When the pressure on the upper side of the valve is higher than the pressure on the bottom side, the differential pressure pushes the cap down to close the vent-hole. Therefore, the non-actuated valve is a pneumatic equivalent of a diode, allowing airflow in one direction only. The minimum pressure required to close the valves in that mode was calculated to be about $1 \mathrm{~Pa}$. On the other hand, when voltage is applied between the top and bottom electrodes, the generated electrostatic pressure pulls the valve cap, thus closing the vent-hole regardless of the differential pressure between the top and bottom sides. A 4-inches wafer-sized skin contains 107 dies, and each dies contains $5 \times 5$ valves. Figure 2 shows the fabrication process flow. We start the process by spinning an AZ4400 photoresist sacrificial layer, approximately $3 \mu \mathrm{m}$ thick. To avoid having solvent bubbles during the subsequent thermal cycles of the process, this photoresist layer needs to be hardbaked at $120^{\circ} \mathrm{C}$ for 45 minutes on a hot plate. Then a $1000 \AA$-thick aluminum layer is evaporated. This layer will be used as an etch-stop during the first etching of parylene, to avoid damaging the sacrificial layer. The first layer of parylene-C is then deposited with a thickness of $4 \mu \mathrm{m}$. To enhance adhesion of the next metal layer, the parylene surface is roughened with low-power oxygen plasma. The bottom 


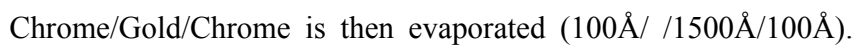
Chrome is used as a buffer layer between parylene and gold to enhance adhesion. After patterning the bottom electrode, the surface is treated with a $5 \%$ A-174 silane solution to improve adhesion between the top chrome and the next parylene. The second layer of parylene $(2 \mu \mathrm{m})$ is then deposited, providing electrical insulation of the bottom electrode (a). A $3500 \AA$ amorphous silicon layer sputtering follows the parylene deposition. This silicon layer is used for anti-stiction purposes [3]. The silicon is patterned a first time and is used as a mask to etch the vent hole into the first and second parylene layers. Parylene etching is done by oxygen plasma. The silicon is then patterned a second time to its final shape (b). A $9 \mu \mathrm{m}$-thick AZ4620 photoresist sacrificial layer is then spun and patterned (c). The third parylene layer $(2 \mu \mathrm{m})$, top electrode $(\mathrm{Cr} / \mathrm{Au} / \mathrm{Cr}: 100 \AA / 1500 \AA / 100 \AA)$, and fourth parylene $(4 \mu \mathrm{m})$ are deposited and patterned (d). The photoresist sacrificial layers are then released in acetone using an ultrasonic bath. The photoresist release is immediately followed by a SAM elf-Assembled Monolayer) coating (e) [4]. This coating makes the

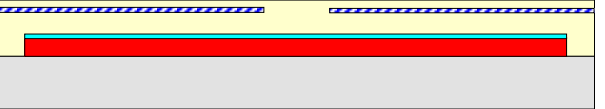

a) Spin PR, evaporate $\mathrm{Al}$, 4um parylene, evaporate $\mathrm{Cr} / \mathrm{Au} / \mathrm{Cr}$ and pattern; 2 um parylene

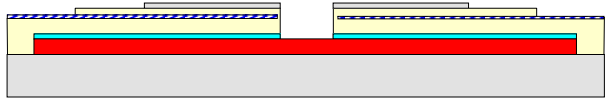

b) Sputter Si and pattern, etch parylene, repattern $\mathrm{Si}$

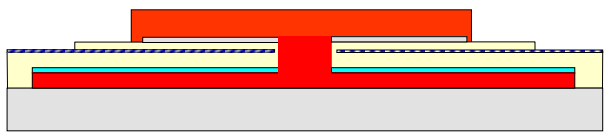

c) Spin thick PR and pattern

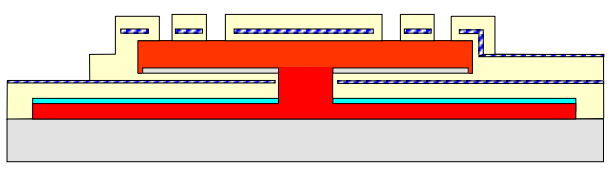

d) 2 um parylene; evaporate $\mathrm{Cr} / \mathrm{Au} / \mathrm{Cr}$ and pattern; 4um parylene and pattern

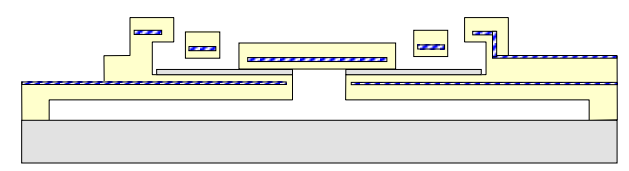

e) Release PR; etch Al, coat SAM

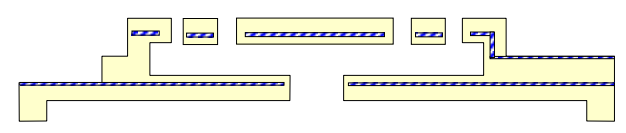

f) BrF3 Si dry etching; cut off the skin from wafer
$\mathrm{Si}$
PR
Parylene
Z $\mathrm{Cr} / \mathrm{Au} / \mathrm{Cr}$

parylene surfaces more hydrophobic, increasing the contact angle from $78^{\circ}$ to $120^{\circ}$ [5]. The samples are then rinsed and dried. At this point of the process some of the valves caps are stuck on the bottom parylene layer (e) due to meniscus force and the low Young's modulus of Parylene (about 4.5GPa). A BrF3 gas is used to etch away the amorophous silicon layer [6], thus releasing the stuck caps (f). The wafer-sized skin is then cut from the wafer (which was unaffected by the whole process and can used for a subsequent run). Figure 3 shows a fabricated skin (4" diameter).

Several challenges had to be overcome during the development of the fabrication process. First, due to the arge difference in linear thermal expansion coefficients of silicon and parylene $\left(2.6^{*} 10^{-6}\right.$ and $3.5^{*} 10^{-5} \mathrm{~K}^{-1}$ respectively) the sputtered silicon film has compressive stress, resulting in wrinkles. To reduce this problem

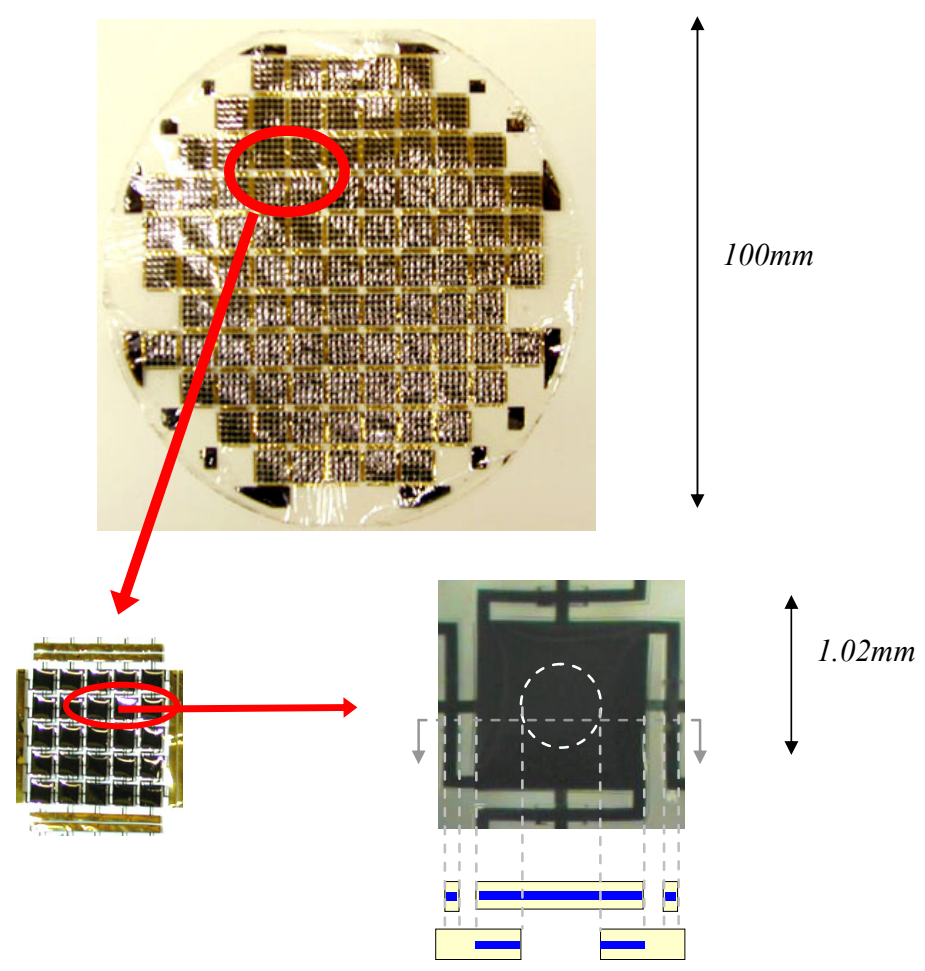

Figure 3: Fabricated Skin, Die close-up and valve close-up

the parylene surface is roughened in low-power oxygen plasma and annealed in a convection oven at $110^{\circ} \mathrm{C}$ for 15 minutes. Lower sputtering power was also found to reduce the compressive stress in the film. Another critical step is the thick sacrificial photoresist layer. The AZ4620 layer has to cover the step made by the venthole otherwise the second and the third parylene layers will fuse together and prevent the valves from opening. Finally, another

\section{Electrostatic Valve Actuators}

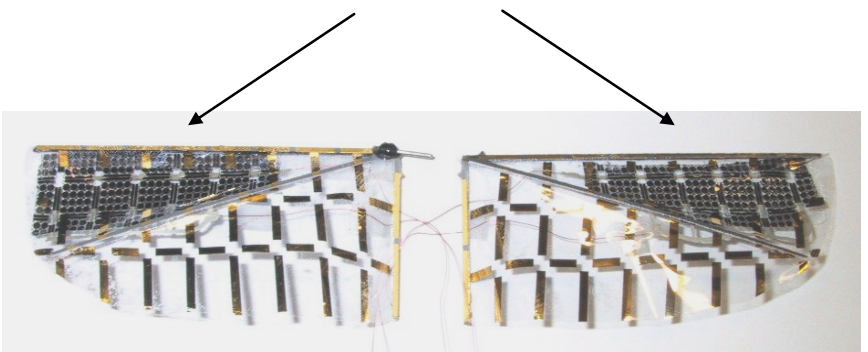

Figure 4: MEMS valved-wing 
processing challenge appeared when etching thick $(>5 \mu \mathrm{m})$ layers of parylene. A black residue of unknown composition tends to be formed on the parylene surface preventing further etching. Reducing the pressure of oxygen (from $400 \mathrm{mT}$ to $200 \mathrm{mT}$ ) can solve this problem.

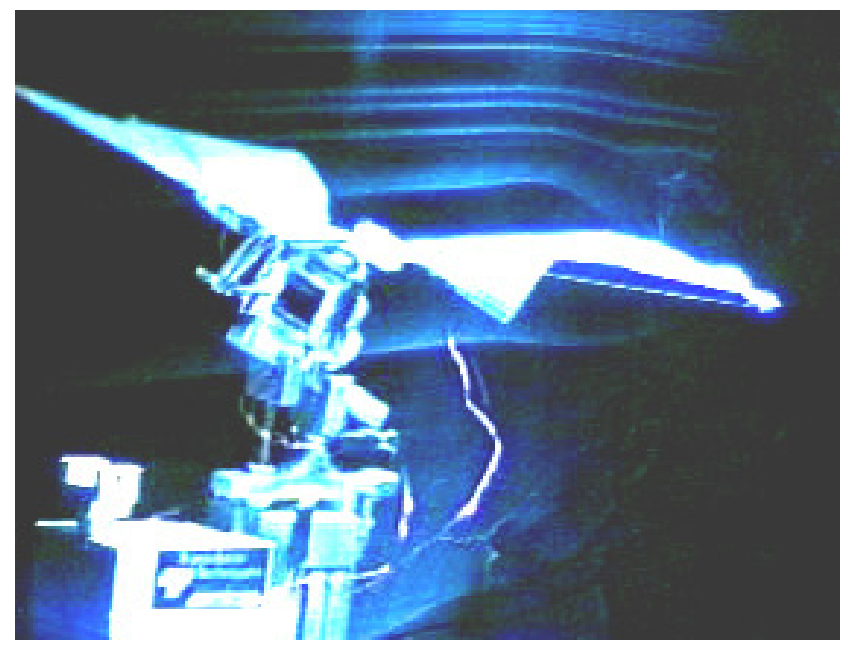

Figure 5: Wind-Tunnel Testing

\section{RESULTS AND DISCUSSION}

First, actuation tests of the valves were performed under microscope. The pull-in voltage was measured to be around $200 \mathrm{~V}$. This is much more than what is expected from a simple theoretical calculation. Several phenomena might be responsible for this. The effective gap is greater than the designed gap, which is given by the AZ4620 layer thickness $(8 \mu \mathrm{m})$. This is due to the flexibility of the skin, which cannot be made perfectly flat. Moreover, stresses in the top electrode results in curling of the valve caps and tethers. Another explanation for this high pull-in voltage could be charging effects in the parylene layers reducing the effective electric field [7].

The valves were tested in a load deflection setup. It was observed that with an applied voltage of $200 \mathrm{~V}$, the valves can withstand a differential pressure of $2 \mathrm{kPa}$ while keeping the vent-holes sealed.

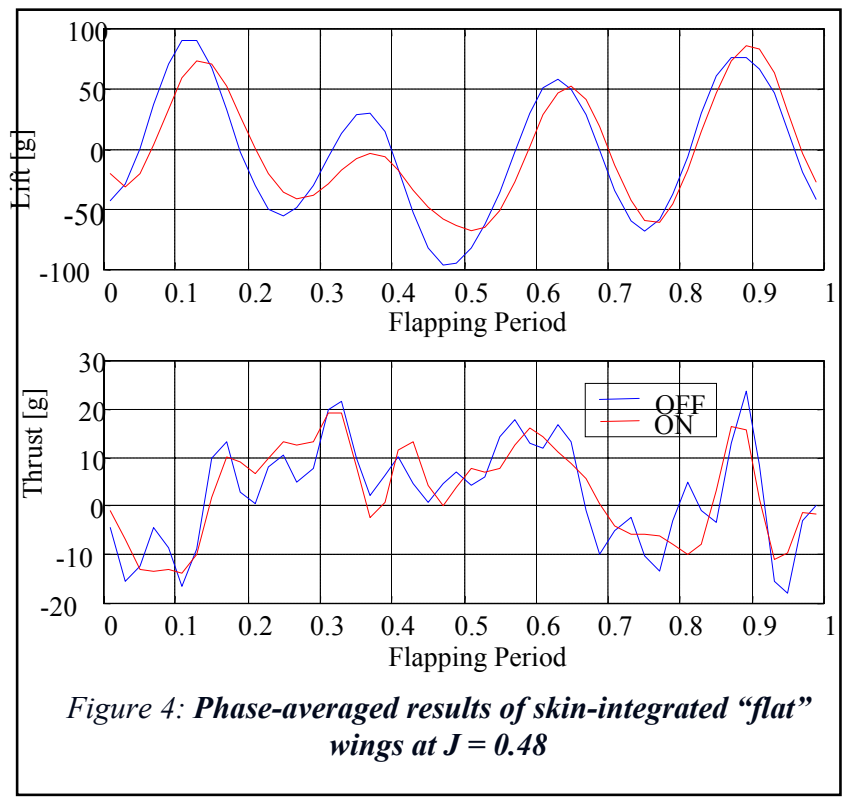

This is much greater than the pressure loading during flapping which is on the order of $40 \mathrm{~Pa}$ The parylene active valves were then integrated onto parylene MEMS wings (about 80 valves per wing) and tested in a low-speed wind tunnel $(0-10 \mathrm{~m} / \mathrm{s})$. The wings were flapped at $20 \mathrm{~Hz}$ by an electric motor while load cells connected to the transmission measure instantaneous lift and thrust. The lift and thrust are piled-up over one single flapping
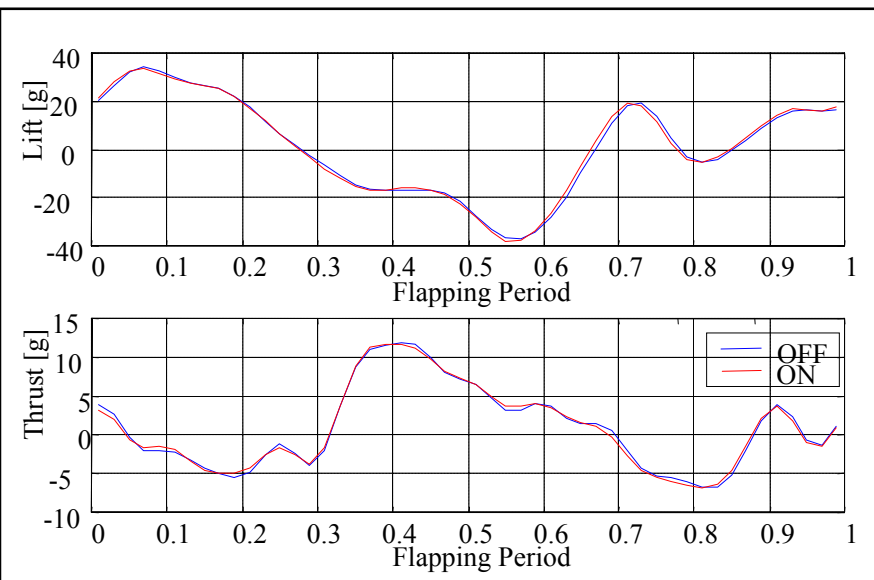

(a) $\mathrm{J}=0.95$
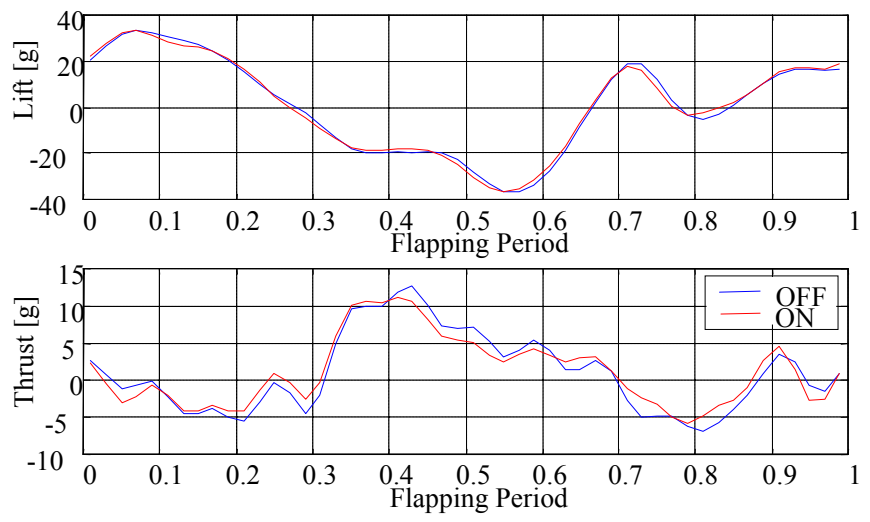

(b) $\mathrm{J}=0.61$
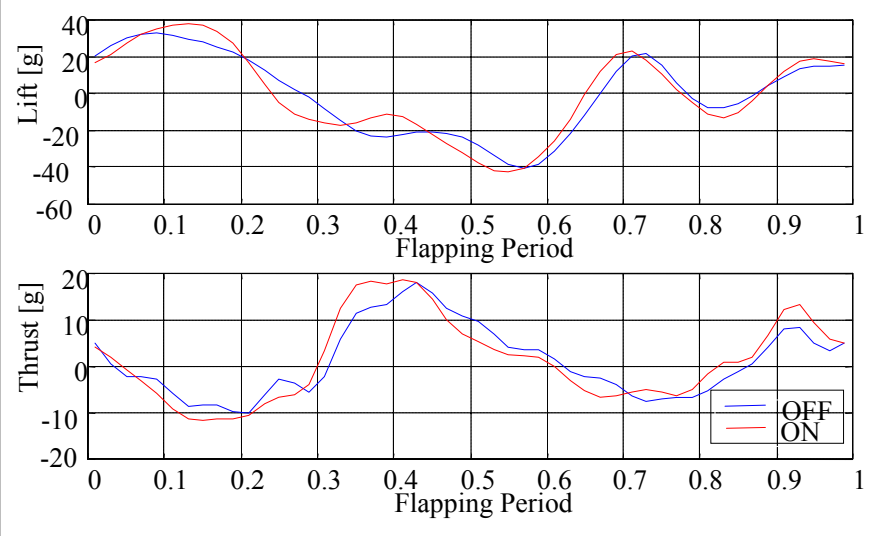

(c) $\mathrm{J}=0.48$

Figure 5: Phase-averaged results of skin-integrated "cambered" wings at various advance ratios. Note that aerodynamic effect is bigger for smaller $J$ 


\begin{tabular}{|c|c|c|}
\hline & ON & OFF \\
\hline Lift $[\mathrm{g}]$ & -1.21 & -1.42 \\
\hline Thrust $[\mathrm{g}]$ & 2.69 & 2.54 \\
\hline
\end{tabular}

Table 1: Time-averaged lift and thrust of skinintegrated "flat" wings at $\mathrm{J}=0.48$

\begin{tabular}{|c|c|c|}
\hline & ON & OFF \\
\hline $\mathrm{J}=0.48$ & 0.14 & -0.01 \\
\hline $\mathrm{J}=0.61$ & 0.99 & 0.94 \\
\hline $\mathrm{J}=0.95$ & 1.43 & 1.42 \\
\hline
\end{tabular}

(a) Lift $[\mathrm{g}]$

\begin{tabular}{|l|l|l|}
\hline & ON & OFF \\
\hline $\mathrm{J}=0.48$ & 1.17 & 1.00 \\
\hline $\mathrm{J}=0.61$ & 1.04 & 0.88 \\
\hline $\mathrm{J}=0.95$ & 0.76 & 0.80 \\
\hline
\end{tabular}

(b) Thrust [g]

\section{Table 2: Time-averaged lift and thrust of skin-integrated “cambered" wings at various advance ratios}

cycle, giving a phase-averaged history. Tests were performed under different advance ratios, angles of attack, and wind speeds. The advance ratio $J$ is a dimensionless number to indicate the unsteadiness of airflow and is given by:

$$
J=\frac{U}{2 \Phi f b}
$$

where $U, f$, and $b$ are wind speed, stroke angle, flapping frequency, and wing length (half wingspan), respectively. $J>1$ indicates an steady regime whereas $J<1$ indicates an unsteady regime. We also investigated the effect of the camber of the wings. Figures 4 and 5 show the phase-averaged lift and thrust histories for flat and cambered wings, with several advance ratios, and with the actuators turned ON and OFF. Tables 1 and 2 give the timeaveraged values of lift and thrust for flat and cambered wings with actuators turned $\mathrm{ON}$ and $\mathrm{OFF}$. The given time-averaged values are obtained by integrating the phase-averaged history over the whole cycle. As can be seen, in every configuration the time-averaged lift and thrust are increased when the actuators are turned on. It is also observed that the effect of the active-valves is greater for more unsteady airflow regime (i.e. lower values of $J$ ). This result was expected because, as explained before, the aerodynamic performances of flapping flight arise from unsteady effects. It is clear that consistent control results have been achieved when the actuators were switched between $\mathrm{ON}$ and OFF states. Up to $18 \%$ of lift and thrust can be gained by actuating the valves when $J=0.45$. Finally, it can be noticed that the valves' effect is more pronounced for cambered wings than for flat wings.

\section{CONCLUSIONS}

We have used our parylene actuators-skin technology to successfully demonstrate the effect of integrated active microvalves on aerodynamic performance of MEMS wings in flapping motion. When used in unsteady airflow regime, the active valves can increase the time-averaged values of lift and thrust by $18 \%$. Future work includes selective actuation of the valves and sensors integration on the skins.

\section{ACKNOWLEDGEMENTS}

This project is supported by DARPA (DABT63-98-C-0005) and the NSF Engineering Research Center at Caltech. Travel support has been generously provided by the Transducers Research Foundation and by the DARPA MEMS and DARPA BioFlips programs.

\section{REFERENCES}

[1] T. N. Pornsin-Sirirak, S.W. Lee, H. Nassef, J. Grasmeyer, Y.-C. Tai, C. -M. Ho, M. Keennon, "MEMS Wing Technology for a Battery-Powered Ornithopter," Proceedings of the $13^{\text {th }}$ IEEE International Conference on MEMS (MEMS'00), Miyazaki, Japan, Jan. 23-27, 2000, pp. 799-804.

[2] T. Nick Pornsin-Sirirak, M. Liger, Y.-C. Tai, S. Ho, C.M. Ho, "Flexible Parylene-Valved Skin for Adaptive Flow Control," ," Proceedings of the $15^{\text {th }}$ IEEE International Conference on MEMS (MEMS '02), Las Vegas, U.S.A., Jan 20-24, 2001, pp101-104

[3] T. J. Yao, X. Yang, and Y. -C. Tai, "BrF ${ }_{3}$ Dry Release Technology for Large Freestanding Parylene MEMS," Digest of Technical Papers of the $11^{\text {th }}$ International Conference on Solid-State Sensors and Actuators (Transducers '01), Munich, Germany, June 10-14, 2001, pp. 652-655.

[4] R. Maboudian, "Self-Assembled Monolayers as AntiStiction Coatings for Surface Microstructures," Digest of Technical Papers, The $10^{\text {th }}$ International Conference on Solid-State Sensors and Actuators (Transducer'99), Vol 1, Sendai, Japan, June 7-10, 1999, pp. 22-25.

[5] T. Nick Pornsin-Sirirak, "Parylene MEMS Technology", $\mathrm{PhD}$ Thesis, California Institute of Technology, 2002, pp 114.

[6] X. Q. Wang, X. Yang, K. Walsh, and Y. C. Tai, "GasPhase Silicon Etching with Bromine Trofluoride," Digest of Technical Papers, The $9^{\text {th }}$ International Conference on Solid-State Sensors and Actuators (Transducers '97), Vol. 2, Chicago, IL, June 16-19, 1997, pp. 1505-1508.

[7] T.J. Yao, K. Walsh and Y.C. Tai,'Dielectrics Charging Effects on Parylene Electrostatic Actuators" Proceedings of the $15^{\text {th }}$ IEEE International Conference on MEMS (MEMS '02), Las Vegas, USA, Jan. 20-24 2002, pp 614617. 